\title{
The Identification and Differentiation between Burkholderia mallei and Burkholderia pseudomallei Using One Gene Pyrosequencing
}

\author{
Damian H. Gilling, Vicki Ann Luna, and Cori Pflugradt \\ Center for Biological Defense, College of Public Health, University of South Florida, Tampa, FL 33612, USA \\ Correspondence should be addressed to Vicki Ann Luna; valuna@cougars.ccis.edu
}

Received 2 April 2014; Revised 11 July 2014; Accepted 15 July 2014; Published 2 October 2014

Academic Editor: Dennis A. Bazylinski

Copyright (C) 2014 Damian H. Gilling et al. This is an open access article distributed under the Creative Commons Attribution License, which permits unrestricted use, distribution, and reproduction in any medium, provided the original work is properly cited.

\begin{abstract}
The etiologic agents for melioidosis and glanders, Burkholderia mallei and Burkholderia pseudomallei respectively, are genetically similar making identification and differentiation from other Burkholderia species and each other challenging. We used pyrosequencing to determine the presence or absence of an insertion sequence IS407A within the flagellin $\mathrm{P}(f l \mathrm{P})$ gene and to exploit the difference in orientation of this gene in the two species. Oligonucleotide primers were designed to selectively target the IS407A-fli $\mathrm{P}$ interface in B. mallei and the fli gene specifically at the insertion point in B. pseudomallei. We then examined DNA from ten B. mallei, ten B. pseudomallei, 14 B. cepacia, eight other Burkholderia spp., and 17 other bacteria. Resultant pyrograms encompassed the target sequence that contained either the fliP gene with the IS407A interruption or the fully intact fliP gene with $100 \%$ sensitivity and $100 \%$ specificity. These pyrosequencing assays based upon a single gene enable investigators to reliably identify the two species. The information obtained by these assays provides more knowledge of the genomic reduction that created the new species B. mallei from B. pseudomallei and may point to new targets that can be exploited in the future.
\end{abstract}

\section{Introduction}

The proteobacteria Burkholderia mallei and Burkholderia pseudomallei, distinct from the Burkholderia cepacia complex, cause melioidosis and glanders, respectively [1]. Inhalation of either organism can lead to pneumonia with reported mortality rates of $19-50 \%[2,3]$. Although human-to-human spread is extremely rare, $B$. mallei is highly infectious when aerosolized and resulting infections can be debilitating, painful, and difficult to diagnose in humans [4]. Historically, B. mallei was used as a bioweapon during the Civil War and the two World Wars with suspected use by the former USSR in Afghanistan [4-6]. Infections with B. pseudomallei are more common especially in endemic areas such as Southeast Asia and Northern Australia [7]. In the United States, rare infections with $B$. pseudomallei have been reported; usually patients are military personnel who were exposed when they were on active duty in endemic areas either recently or even as long as 62 years before $[8,9]$. Two active disease cases were reported in US civilians who had traveled to Honduras where they presumably were exposed [10] (CDC, 2006). Other cases have been reported in Arizona, Puerto Rico, and the British Virgin Islands as recently as 2011 where the source of infection appeared to be local soils and flood waters [11-15]. Laboratory exposure has also been reported in California and Florida [10, 16]. Today, both B. mallei and B. pseudomallei are classified as Tier 1 select agents by the Centers for Disease Control and Prevention (CDC) due to their high virulence via the respiratory route, as well as potential to cause illness on a large scale if ever widely disseminated [6].

Symptoms of B. pseudomallei infection vary, ranging from severe pneumonia with concomitant abscesses in the liver and spleen to persistent infections of the skin, soft tissues, bones, and joints [2]. Often the symptomology of $B$. pseudomallei infections parallel to those of infections such as tuberculosis which makes empiric diagnosis difficult unless the organism can be isolated, identified, and confirmed in the laboratory $[2,17,18]$. Another factor which makes the clinical 
identification and differentiation between B. pseudomallei and $B$. mallei even more challenging is the fact that human cases of glanders and melioidosis infections are not common in nonendemic parts of the world. As a result many medical professionals in clinics and laboratories lack experience with these bacteria [18, 19]. Additionally, B. pseudomallei has earned the nickname "the great mimicker" due to its wide repertoire of clinical manifestations which often prompt misdiagnosis. Several rapid detection/diagnostic biochemical methods have been introduced; yet problems have been reported such as the misidentification of $B$. pseudomallei as B. cepacia [19-23]. Thus research has shifted focus toward molecular methods, but these too have had their problems, primarily assay specificity and sensitivities which are less than $100 \%[24,25]$. Culture methods have traditionally been the gold standard for identifying B. mallei and B. pseudomallei $[26,27]$. However these methods can be time consuming. Many molecular methods focus on $B$. mallei and the loss of a gene or phenotype and appear to be reliable in identifying $B$. mallei but none of these methods can consistently distinguish B. pseudomallei from other Burkholderia species [19, 25]. DNA fingerprinting assays such as multilocus sequence typing (MLST) have been proven efficacious at detection but are also time consuming, expensive, and technically challenging for many laboratories [28]. Identification of B. mallei and $B$. pseudomallei through the sequencing of 16S DNA targets has been successfully accomplished; however, discrimination of the two closely related species requires additional sequencing $[29,30]$.

Genetically, B. pseudomallei and B. mallei are so similar that differentiating between the two is a challenge. In fact B. mallei evolved directly from $B$. pseudomallei due to a process called reductive evolution wherein a massive invasion of many insertion sequence (IS) elements altered the $B$. pseudomallei genome through genomic rearrangements and deletions. This reduction evolution resulted in a smaller genome with enough key genotypic and phenotypic differences to create a separate species now known as $B$. malle $i$ $[31,32]$. The numerous $I S$ elements in the $B$. mallei genome account for about $3.1 \%$ of the total genome; yet this smaller genome is only $80 \%$ in size of that of $B$. pseudomallei $[32,33]$. One specific insertion sequence, known as IS407A can be located in multiple sites in the genome and has been found to interrupt the flagellar gene $(f l i \mathrm{P})$ that encodes the flagellar $P$ protein and renders $B$. mallei nonmotile [33]. Additionally, the resultant truncated fliP gene is completely flipped, having undergone a complete inversion on the DNA strand. This genomic structure and nonmotility is found in all $B$. mallei [32]. In comparison, $B$. pseudomallei does not contain the IS407A insertion sequence within its fliP gene. This complete gene confers full motility and contains sequence regions that are distinct from the fli $\mathrm{P}$ gene in other Burkholderia species such as B. cepacia.

The B. mallei fliP/IS407A region with its species wide conserved gene inversion and truncation provided the target for $B$. mallei used in this work. We hypothesized that when the target region was sequenced, that sequence would exhibit both the end of the IS407A insertion and a section of the fliP gene that would be in the reverse orientation to that same gene found in $B$. pseudomallei. We then chose a $B$. pseudomallei target that covered the same section of the fliP gene where the IS407A insertion sequence was located in $B$. mallei, using the rationale that, when sequenced, this target will exhibit either (A) a complete fli $\mathrm{P}$ match in the correct orientation and thus can be identified as B. pseudomallei or (B) nothing at all if the gene is interrupted which precluded the primers from binding. Thus this scheme is able to detect and differentiate between B. pseudomallei and B. mallei. We hypothesized that the targeting of the fliP gene with its clear differences between B. mallei and B. pseudomallei and distinctions from other Burkholderia spp. should provide a reliable form of confirmation between inconclusive results.

The purpose of this study was to explore the use of pyrosequencing to exploit the genetic difference of the fliP gene, its orientation, and surrounding sequences in $B$. mallei and $B$. pseudomallei as a single gene target method of detection and differentiation between the two species and other Burkholderia species. We wanted to determine if the pyrosequencing platform, that is, putatively simple, quick, and trustworthy [34], would enable the consistent and reliable microbial typing by targeting conserved fli $\mathrm{P}$ regions of $B$. pseudomallei in addition to the variable fli $\mathrm{P} / I S 407 \mathrm{~A}$ regions of B. mallei.

\section{Materials and Methods}

2.1. Bacterial Strains. We examined ten Burkholderia mallei, ten Burkholderia pseudomallei, and 40 other bacterial strains, including $15 \mathrm{~B}$. cepacia, $3 \mathrm{~B}$. thailandensis, and five various Burkholderia species (Table 1). The B. mallei, B. pseudomallei, and $2 B$. thailandensis were received from the NIH Biodefense and Emerging Infections Research Resources Repository (BEI Resources, Bethesda, MD, USA) as either live strains or purified DNA. Access to both isolates and DNA of both $B$. mallei and $B$. pseudomallei were limited due to restrictions on how many Tier 1 isolates and DNA can be purchased yearly from BEI Resources. Of the $15 \mathrm{~B}$. cepacia isolates, one was obtained from the American Type Culture Collection (ATCC, Manassas, VA, USA), three were collected from the Florida Department of Health, Bureau of Laboratories, Tampa, FL (FDOH) and 11 were procured from the University of Washington Medical Center in Seattle, WA. The other 23 bacterial strains including six various Burkholderia species were part of our large bacterial collection and were either collected previously as clinical isolates from FDOH or received from ATCC (Table 1).

All manipulations of cultures of B. mallei and B. pseudomallei strains were performed in a biological safety cabinet in a biological safety level 3 (BSL3) laboratory. All safety protocols followed "Biosafety in Microbiological and Biomedical Laboratories, 5th Edition" (BMBL) practices including the use of protective laboratory clothing and respiratory equipment [35]. The safety and security requirements by US federal regulation DHHS 42 CFR 73 were strictly adhered to. Manipulations of the other bacterial strains were performed in a biological safety level 2 (BSL2) laboratory in a biological safety cabinet following safety practices outlined in BMBL as above. 
TABLE 1: List of bacteria used in this study.

\begin{tabular}{|c|c|c|}
\hline Organism & CBD number $^{\mathrm{a}}$ & Historical strain identifier \\
\hline \multicolumn{3}{|l|}{ Burkholderia } \\
\hline Burkholderia mallei & BB 0044 & China 5 (MM-A, NBL 4) ATCC 10399 \\
\hline Burkholderia mallei & BB 0045 & Ivan (NCTC 10230) ATCC 15310 \\
\hline Burkholderia mallei & BB 0046 & China 7 (NBL 7) ATCC 23344 \\
\hline Burkholderia mallei & BB 0050 & GB8 horse 4 \\
\hline Burkholderia mallei & DD-675 & BURK014 \\
\hline Burkholderia mallei & DD-671 & BURK005 (SR092700A) \\
\hline Burkholderia mallei & DD-372 & BURK007 (SR092700C) \\
\hline Burkholderia mallei & DD-673 & BURK009 (SR092700E) \\
\hline Burkholderia mallei & DD-677 & BURK062 (Turkey 1) \\
\hline Burkholderia mallei & DD-178 & BURK010 (2344) \\
\hline Burkholderia pseudomallei & BB 0047 & China 3 (MP-H, NBL 104) \\
\hline Burkholderia pseudomallei & BB 0048 & S 397 (NRRL B-1112, CCEB 472) \\
\hline Burkholderia pseudomallei & BB 0049 & 286 (MP-S, NBL 121) \\
\hline Burkholderia pseudomallei & BB 0051 & K96243 \\
\hline Burkholderia pseudomallei & BB 0052 & $1026 b$ \\
\hline Burkholderia pseudomallei & BB 0053 & $1106 \mathrm{~b}$ \\
\hline Burkholderia pseudomallei & BB 0054 & $1710 \mathrm{a}$ \\
\hline Burkholderia pseudomallei & BURK088 & BURK088 \\
\hline Burkholderia pseudomallei & BURK099 & BURK099 \\
\hline Burkholderia pseudomallei & NR-9320 & K96423 \\
\hline Burkholderia cepacia & CBD 1341 & FL-M-05-0506072 \\
\hline Burkholderia cepacia & CBD 1342 & FL-M-05-B24210 \\
\hline Burkholderia cepacia & CBD 1343 & FL-M-05-B25995R \\
\hline Burkholderia cepacia & CBD 1442 & ATCC BAA-245 \\
\hline Burkholderia cepacia & CBD 1450 & F68492 \\
\hline Burkholderia cepacia & CBD 1456 & H27659 \\
\hline Burkholderia cepacia & CBD 1455 & H35975 \\
\hline Burkholderia cepacia & CBD 1449 & M25311 \\
\hline Burkholderia cepacia & CBD 1458 & M27066 \\
\hline Burkholderia cepacia & CBD 1451 & M42544 \\
\hline Burkholderia cepacia & CBD 1453 & M52455 \\
\hline Burkholderia cepacia & CBD 1454 & M74393 \\
\hline Burkholderia cepacia & CBD 1457 & $\mathrm{~T} 10400$ \\
\hline Burkholderia cepacia & CBD 1452 & T33589 \\
\hline Burkholderia cepacia & CBD 1448 & T47491 \\
\hline Burkholderia graminis & CBD 1440 & ATCC 700544 \\
\hline Burkholderia multivorans & CBD 1443 & ATCC BAA-247 \\
\hline Burkholderia stabilis & CBD 1441 & ATCC BAA-67 \\
\hline Burkholderia vietnamiensis & CBD 1438 & ATCC 55792 \\
\hline Burkholderia vietnamiensis & CBD 1444 & ATCC BAA-248 \\
\hline Burkholderia thailandensis & CBD 1439 & ATCC 700388 \\
\hline Burkholderia thailandensis & BURK254 & E254 \\
\hline Burkholderia thailandensis & BURK43 & MSMB043 \\
\hline \multicolumn{3}{|l|}{ Other bacteria } \\
\hline Achromobacter xylosoxidans & CBD 0307 & ATCC 27061 \\
\hline Acinetobacter baumannii & CBD $1323^{b}$ & ATCC 19606 \\
\hline Acinetobacter calcoaceticus & CBD $1336^{b}$ & ATCC 23055 \\
\hline Bacillus badius & CBD 0091 & ATCC 6462 \\
\hline Cedecea neteri & CBD 0309 & ATCC 33855 \\
\hline Citrobacter freundii & CBD $0553^{\mathrm{b}}$ & ATCC 8090 \\
\hline
\end{tabular}


TABle 1: Continued.

\begin{tabular}{lcc}
\hline Organism & CBD number $^{\mathrm{a}}$ & Historical strain identifier \\
\hline Enterobacter cloacae & CBD $0556^{\mathrm{b}}$ & ATCC 13047 \\
Enterococcus faecalis & CBD 1406 & ATCC 49532 \\
Enterococcus faecalis & CBD 1407 \\
Klebsiella pneumoniae & CBD 0555 & ATCC 49533 \\
Lactobacillus rhanmosus & CBD 1409 & ATCC 35657 \\
Pseudomonas aeruginosa & CBD 0551 & ATCC 53103 \\
Serratia marcescens & CBD 1239 & ATCC 15442 \\
Staphylococcus aureus & CBD 0534 & ATCC 13880 \\
Staphylococcus aureus & CBD 1276 & WA-HMC-03-4905 \\
Stenotrophomonas maltophilia & CBD 0552 & ATCC BAA-976 \\
Streptococcus pneumoniae & CBD 1405 & ATCC 51331 \\
\hline
\end{tabular}

${ }^{a} \mathrm{CBD}$ : CBD stands for "Center for Biological Defense" and is the starting designation we use for bacterial strains that are in our BSL2 bacterial collection. Besides the CBD letter designation, each strain in our collection is also given a number to identify it. Each isolate in our BSL3 bacterial collection has a BB letter designation and then assigned a number as the BSL2 strains.

${ }^{\mathrm{b}}$ denotes an isolate carries a homolog of the fliP gene.

2.2. DNA Extraction. Bacteria were grown on tryptic soy agar supplemented with $5 \%$ sheep red blood cells (blood agar (BA)) (Remel, Lenexa, KS, USA). All culture plates were incubated at $35^{\circ} \mathrm{C}$ overnight (18-24 hours) before performing DNA extractions. Following manufacturers' instructions, all genomic DNA extractions were either performed using the Epicentre (Qiagen Inc, Madison, WI) extraction kits, a MagNaPure Compact automated instrument (Roche, Inc, Indianapolis, IN, USA), or a boil preparation method used by $\mathrm{FDOH}$. The boil preparation, (adapted from a reference lab in the Laboratory Response Network) is described as follows: bacterial growth from an overnight cultured media plate was removed and placed into $100 \mu \mathrm{L}$ of sterile water and boiled for 5 minutes and then placed onto ice for 2 minutes, followed by centrifugation at $12,000 \times \mathrm{g}$ for 10 minutes at $4^{\circ} \mathrm{C}$. The supernatant was transferred to a $0.1 \mu \mathrm{M}$ filter tube (Millipore Corporation Billerica, MA) and centrifuged for 2 minutes at $8,000 \times \mathrm{g}$. To prove sterility of all filtrates and DNA extractions (following the University of South Florida Institutional Biosafety Committee guidelines), $10 \mu \mathrm{L}$ was sacrificed and used to inoculate a BA media culture plate and incubated at $35^{\circ} \mathrm{C}$ for 48 hours. Extracts and lysates having no growth after two days were allowed out of the BSL3 environment and made available for molecular work. The DNA was stored at $4^{\circ} \mathrm{C}$ or $-30^{\circ} \mathrm{C}$ until used. Before use, the DNA was diluted 1:20 in molecular-grade water (Fisher Scientific, Pittsburg, PA) to achieve a working concentration of 0.5 to $2 \mathrm{ng} / \mu \mathrm{L}$.

2.3. Validation PCR Assays and Gel Electrophoresis. Based upon the fliP gene GenBank sequence BMA2686, PCR assays were designed to target sequences of the fli $\mathrm{P}$ gene in B. mallei (NCBI GenBank Accession \#:NC_006348) and B. pseudomallei (NCBI GenBank Accession \#:NC_009076). Figure 1 shows a schematic of the target regions in both species. All oligonucleotides used in the PCR assays for $B$. mallei and B pseudomallei are listed in Table 2. Primer-BLAST analysis (via NCBI) predicted $217 \mathrm{bp}$ and $397 \mathrm{bp}$ amplicons for the fliP-IS407A interface in B. mallei and the fliP gene in
B psudomallei, respectively. The PCR reaction mixture with a final volume of $10 \mu \mathrm{L}$ volume consisted of $1 \mu \mathrm{L}$ of DNA template (at a concentration of $0.5-2 \mathrm{ng} / \mu \mathrm{L}$ ), $1 \mu \mathrm{L}$ of the Takara (Takara, Madison, WI, USA) 10X buffer containing $1.5 \mathrm{mM}$ $\mathrm{MgCl}_{2}, 0.82 \mu \mathrm{L}$ of dNTPs at $25 \mu \mathrm{M}, 0.05 \mu \mathrm{L}$ of Takara HS Taq enzyme, and $2 \mu \mathrm{L}$ of each specific primer at a concentration of $1 \mu \mathrm{M}$. Universal primers for the $16 \mathrm{~S}$ rDNA sequence of Burkholderia spp. previously described [20, 30] were later incorporated in the assays and produced a $1500 \mathrm{bp}$ product to act as a control. The universal primers were IAC-16SF ( $5^{\prime}$-AGAGTTTGATCCTGGCTCAG- $\left.{ }^{\prime}\right)$ and IAC-rDNA-R $\left(5^{\prime}\right.$-ACGGCTACCTTGTTACGACTT-3 $\left.{ }^{\prime}\right)$. All PCR reactions were carried out on a T1 Biometra Thermocycler (Biometra Inc., Göttingen, Germany) with the following conditions: initial denaturation at $95^{\circ} \mathrm{C}$ for 15 minutes, followed by 35 cycles of $94^{\circ} \mathrm{C}$ for 30 seconds, $58^{\circ} \mathrm{C}$ for 1 minute, and $72^{\circ} \mathrm{C}$ for 3 minutes, and lastly a final extension of $72^{\circ} \mathrm{C}$ for 10 minutes.

PCR amplicons were electrophoresed on a $1 \%$ agarose gel containing $15 \mu \mathrm{g}$ of ethidium bromide (final concentration of $0.2 \mu \mathrm{g} / \mathrm{mL})$ in $1 \mathrm{X}$ TBE $(44.5 \mathrm{mmol}$ Tris-borate and $1 \mathrm{mmol}$ EDTA, pH 8.3) for $60 \mathrm{~min}$ at $100 \mathrm{mV}$ constant voltage. DNA bands were visualized with UV light and photographed using the GelDoc (Bio-Rad, Hercules, CA, USA).

2.4. Sanger Sequencing. The amplicons from the validation PCR and the initial pyrosequencing PCR reactions were purified using the Wizard DNA Purification Kit (Promega, Madison, WI, USA). Cycle sequencing was performed using the Beckman-Coulter Dye Terminator Cycle Sequencing (DTCS) Quick Start kit protocol (Beckman-Coulter, Fullerton, CA, USA). Cycle sequencing reactions with a final volume of $20 \mu \mathrm{L}$ consisted of $8 \mu \mathrm{L}$ DTCS Quick Start master mix (Beckman-Coulter), $1.5 \mu \mathrm{L}$ sequence primer at $25 \mathrm{pmol}$, and $8 \mu \mathrm{L}$ of DNA. Cycle sequencing PCR was performed with the Biometra T3 Thermocycler (Biometra Inc.)) for 30 cycles under the following conditions: $96^{\circ} \mathrm{C}$ for 20 seconds, $50^{\circ} \mathrm{C}$ for 20 seconds, and $60^{\circ} \mathrm{C}$ for 4 minutes. The DNA produced from the cycle sequencing reaction was precipitated as per the Beckman-Coulter ethanol precipitation protocol. Finally, 
TABLE 2: List of oligonucleotide primers used in pyrosequencing assays.

\begin{tabular}{|c|c|c|c|c|}
\hline Organism & Target & Primer & Sequence $\left(5^{\prime}-3^{\prime}\right)$ & Location $(\mathrm{bp})^{\mathrm{a}}$ \\
\hline \multirow{4}{*}{ B. mallei } & fliP & $\mathrm{BmfliP}_{\mathrm{F}} \mathrm{b}^{\mathrm{b}}$ & ACGAACAGCGTGAGGAAGAG & $2786291-2786310$ \\
\hline & $I S 407 \mathrm{~A}$ & BmIS407A-R1 & CTAGAAGCCCATTGGCCCTAT & $2786443-2786423$ \\
\hline & Interface & $\mathrm{Bm}-\mathrm{S} 1$ & GGGGCAGGTCAACGA & $2786417-2786403$ \\
\hline & Interface & $\mathrm{Bm}-\mathrm{S} 3$ & GGCAGGTCAACGAGC & $2786415-2786401$ \\
\hline \multirow{3}{*}{ B. mallei } & $f l i \mathrm{P}$ & BmfliP-F2 $^{b}$ & CGAACAGCGTGAGGAAGAG & $2786292-2786310$ \\
\hline & IS407A & BmIS407A-R1 & CTAGAAGCCCATTGGCCCTAT & $2786443-2786423$ \\
\hline & Interface & $\mathrm{Bm}-\mathrm{S} 3$ & GGCAGGTCAACGAGC & 2786415-2786401 \\
\hline \multirow{4}{*}{ B. pseudomallei } & $f l i \mathrm{P}$ & BpfliP-F1 & AGACGATGCTGCTGCTCAC & $31112-31130$ \\
\hline & $f l i \mathrm{P}$ & BpfliP-R1 ${ }^{\mathrm{b}}$ & CCCGACGAGCACCTGATTC & $31257-31239$ \\
\hline & fli $\mathrm{P}$ & BpfliP-R2 ${ }^{b}$ & GAACAGCGTGAGGAAGAGGG & $31281-31262$ \\
\hline & $f l i \mathrm{P}$ & BpfliP-S4 & GCTGTCGTTCCTGCC & $31134-31148$ \\
\hline \multirow{3}{*}{ B. pseudomallei } & fliP & BpfliP-F2 ${ }^{b}$ & GACGATGCTGCTGCTCAC & $31113-31130$ \\
\hline & $f l i \mathrm{P}$ & BpfliP-R3 & AACAGCGTGAGGAAGAGGG & $31280-31262$ \\
\hline & fli $\mathrm{P}$ & BpfliP-S5 & AGCAGCGACAGCACG & $31205-31191$ \\
\hline
\end{tabular}

Pyrosequencing primer sets for B. mallei and B. pseudomallei with respective target regions. Primers were designed using the design software supplied by the manufacturer.

a denotes location on the published sequences of either B. mallei GenBank accession number NC_006348 or B. pseudomallei GenBank accession number NC_009076.

${ }^{\mathrm{b}}$ denotes a biotinylated primer.

"Interface" denotes the sequence where IS407A interrupts the fliP gene.

"F" denotes forward primer used for PCR.

"R" denotes reverse primer used for PCR.

"S" denotes the pyrosequencing primers.

The primers were first rehydrated in molecular grade water to bring them to a $100 \mu \mathrm{M}$ concentration for each primer. Forward and reverse primers were used at $1 \mu \mathrm{M}$ concentration while the sequence primers were used at $100 \mu \mathrm{M}$.

The target sequence for the primer sets for $B$. mallei was: GCCTGCCGCAGCAGCGACAGCACGACGATGATCCGCGTGA, located at 2786360-2786399 bp in the sequence NCBI Genbank Accession \#: NC_006348. The target sequence for the primer sets for B. pseudomallei was: GCGATGCTGCTGATGATGACGAGCTTCACGCGGATCATCA, located in the NCBI Genbank Accession \#: NC_009076 at 31150-31189 bp. PCR primer targets for both B. mallei and B. pseudomallei are all located on their respective chromosome 1. All primer coordinates were last verified on April 1, 2014.

Sanger sequencing was performed on a Beckman-Coulter CEQ-8000 (Fullerton, CA) following the manufacturer's directions. The resulting nucleotide sequences were compared to the public databases via NCBI nucleotide nBLAST alignment which confirmed or negated the sequences' identity for either B. mallei or B. pseudomallei.

2.5. Pyrosequencing. Initial pyrosequencing PCR assays with the validation primers described above for $B$. mallei and $B$. pseudomallei were carried out in $50 \mu \mathrm{L}$ reaction volumes. The amplicons were then used as template for the pyrosequencing assays using primers that nested within the amplicon sequence (Table 2 and Figure 1). One primer from each primer set carried a biotin tag at the $5^{\prime}$ end (Table 2). Different primer sets were used to examine if the target sequence could be produced consistently or if some primer locations were better than others. Finally, sepharose beads were used to capture the remaining biotinylated PCR product, after which the product-bead mixture was washed using a series of solutions that removed impurities and stripped the double-stranded DNA down to single-stranded DNA. This was done using the Pyromark vacuum prep worktable (Qiagen, Valencia, CA, USA). Specific pyrosequencing primers (Table 2) designed to target the regions of interest on B. mallei and B. pseudomalle $i$ were then annealed to the single-stranded DNA template before analysis on the Q96 ID Pyrosequencer (Qiagen).
This procedure was performed as per the manufacturer's instructions with each sample sequenced at least twice. The assay was conducted in the sequence analysis (SQA) mode and the pyrosequencing results were graded as pass or fail based on the test samples' adherence to the predetermined qualifications as per the manufacturer's directions. All strains were tested a minimum of two times to confirm results.

\section{Results and Discussion}

In the validation PCR assays, the fli $\mathrm{P}$ target regions of $B$. mallei and $B$. pseudomalle $i$ were successfully amplified. The fliP target gene region for $B$. mallei produced a $217 \mathrm{bp}$ PCR product as predicted while the assay for the $B$. pseudomallei target region produced a $397 \mathrm{bp}$ amplicon (data not shown). All ten B. mallei amplicons from the PCR assays when sequenced using the Sanger sequencing method had $100 \%$ nucleotide identity with the target sequence (B. mallei fliP gene and insertion sequence in GenBank accession NC_006348). When sequenced using the Sanger sequencing method, all ten amplicons produced from the B. pseudomallei DNA templates displayed $100 \%$ nucleotide identity with the published target gene (B. pseudomallei fliP gene sequence GenBank accession NC_009076). B. cepacia which possesses its own version of the fliP gene per published GenBank sequences have little to no nucleotide identity for either 


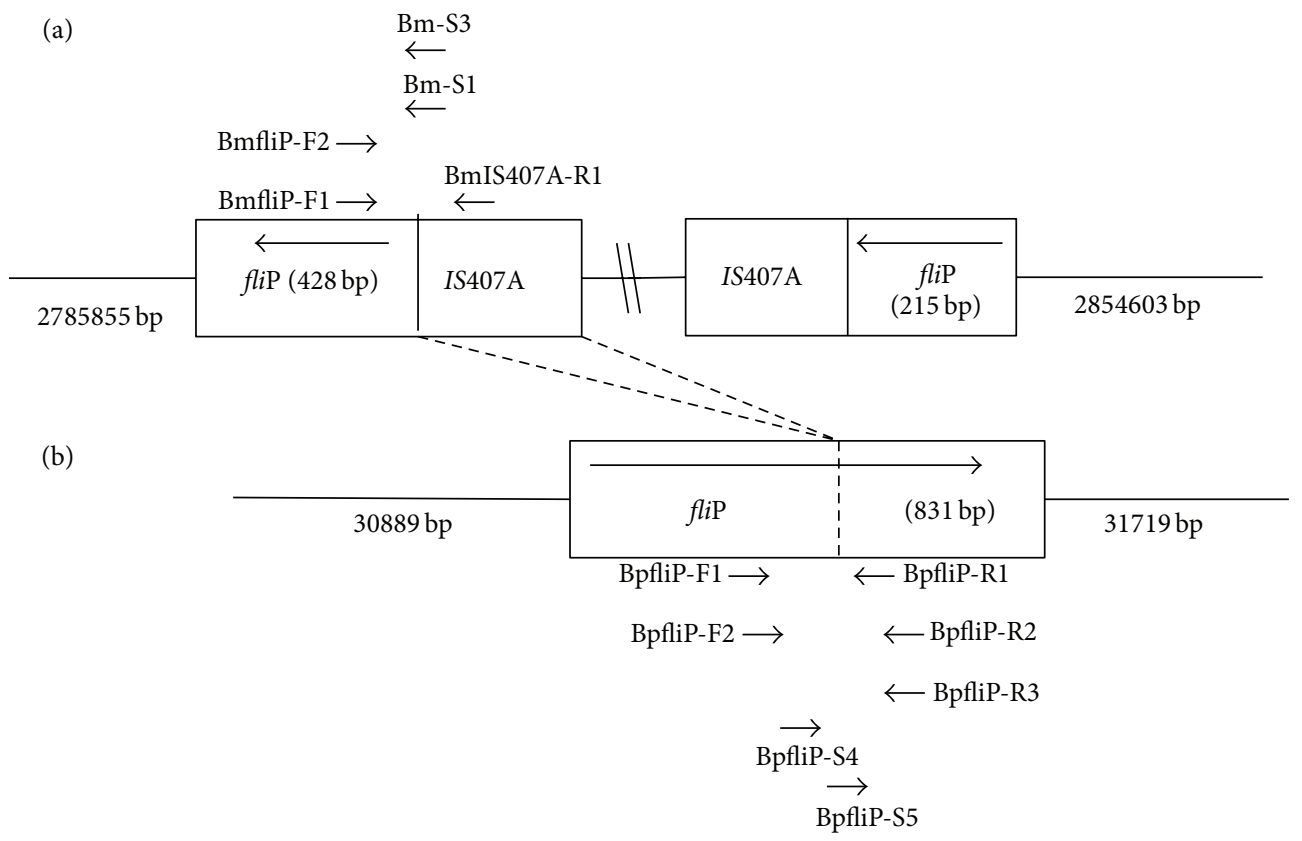

FIGURE 1: (a) Schematic of Burkholderia mallei fliP gene with IS407A interruption that caused both gene inversion and loss of function. The large arrow within the block designates the orientation of the two parts of the fliP gene on the DNA strand. The location and direction of the various primers are noted by the small arrows. The pyrosequencing target in B. mallei is the interface between the insertion sequence element and fliP gene. (b) Schematic of the Burkholderia pseudomallei intact and functional fliP gene. The large arrow within the block designates the orientation of the intact gene while the location and directions of the primers used are denoted by the small arrows. The pyrosequencing target in B. pseudomallei covers both sides of the point of the fliP gene that corresponds to where the insertion sequence is found in $B$. mallei.

B. mallei or B. pseudomallei primers. As predicted none of the B. cepacia DNA templates nor the DNA from the six various Burkholderia spp. produced amplicons when used in either of the two PCR assays. Additionally, the DNA templates from the remaining bacterial strains, including the seven bacterial collection strains which possess their own versions of the fliP genes, yielded negative results.

When the DNA from the ten B. mallei strains were pyrosequenced with $B$. mallei pyrosequencing primers (Table 2) all ten tests were noted as "PASSED" and the resulting pyrograms were confirmed via NCBI nucleotide nBLAST alignment (Table 3). The NCBI nBLAST alignments confirmed that the pyrosequencing sequences matched with $100 \%$ nucleotide identity to publicly available fliP sequences of $B$. mallei. Different combinations of primer sequences (Table 2) were utilized to explore a larger target sequence and also resulted in $100 \%$ nucleotide identity no matter if the target was shifted slightly up or down stream (Figure 1). When the DNA from the ten $B$. mallei strains were pyrosequenced with the B. pseudomallei pyrosequencing primers (Table 2), all of the pyrograms were noted as "FAILED." When the DNA from the ten $B$. pseudomallei strains were pyrosequenced using the B. pseudomallei sequencing primers (Table 2), all ten were noted as "PASSED" and when tested with the B. mallei pyrosequencing primers they were noted as "FAILED." The resulting "PASSED" pyrograms were confirmed via NCBI nucleotide nBLAST and again matched with $100 \%$ nucleotide identity to published $B$. pseudomallei fli $\mathrm{P}$ sequences. The various primer sets that were designed and used (Table 2) generated sequences that shifted slightly up and down stream (Figure 1). The average pyrosequencing result obtained for the $B$. pseudomallei DNA templates was 44 nucleotides long (like the B. mallei results) and all had $100 \%$ nucleotide identity to $B$. pseudomallei GenBank sequences of the fliP gene and not for fliP gene sequences of other Burkholderia species. Repeated testing gave the same results. As displayed in Table 3 the pyrosequencing results for B. mallei and B. pseudomallei show the highest percentage matches when applied to an NCBI BLAST search. Both assays demonstrated $100 \%$ sensitivity as they reliably and consistently identified the known $B$. mallei or $B$ pseudomallei DNA. This pyrosequencing method also demonstrated $100 \%$ specificity against the DNA samples of 15 B. cepacia and six other Burkholderia spp. All yielded negative PCR results and failed pyrosequencing readouts with both of the $B$. mallei and B. pseudomallei pyrosequencing primer sets (Table 4). All pyrosequencing nucleotide sequences will be submitted for entry into the NCBI databases.

Reliable detection and differentiation of B. mallei and B. pseudomallei continue to be challenging because of the difficulty in developing a consistently sensitive, selective, and accurate assay [25]. Current molecular techniques such as ribotyping, and multilocus sequence typing are employed for detection and differentiation, but these methods can be labor intensive and challenging for laboratories and their usefulness is questionable in distinguishing differences between B. mallei and B. pseudomallei $[19,28]$. Here we present 
TABLE 3: Results of NCBI BLASTs of Sequences obtained by Pyrosequencing Assay.

\begin{tabular}{lcc}
\hline Organism name & CBD Number & NCBI GenBank accession number \\
\hline Burkholderia mallei & BB 0044 & CP000548.1 Burkholderia mallei \\
Burkholderia mallei & BB 0045 & CP000548.1 Burkholderia mallei \\
Burkholderia mallei & BB 0046 & CP000548.1 Burkholderia mallei \\
Burkholderia mallei & BB 0050 & CP000548.1 Burkholderia mallei \\
Burkholderia mallei & DD-675 & CP000548.1 Burkholderia mallei \\
Burkholderia mallei & DD-671 & CP000548.1 Burkholderia mallei \\
Burkholderia mallei & DD-372 & CP000548.1 Burkholderia mallei \\
Burkholderia mallei & DD-673 & CP000548.1 Burkholderia mallei \\
Burkholderia mallei & DD-677 & CP000548.1 Burkholderia mallei \\
Burkholderia mallei & DD-178 & CP000548.1 Burkholderia mallei \\
Burkholderia pseudomallei & BB 0047 & CP006470.1 Burkholderia pseudomallei \\
Burkholderia pseudomallei & BB 0048 & CP006470.1 Burkholderia pseudomallei \\
Burkholderia pseudomallei & BB 0049 & CP003781.1 Burkholderia pseudomallei \\
Burkholderia pseudomallei & BB 0051 & CP006470.1 Burkholderia pseudomallei \\
Burkholderia pseudomallei & BB 0052 & CP003781.1 Burkholderia pseudomallei \\
Burkholderia pseudomallei & BB 0053 & CP006470.1 Burkholderia pseudomallei \\
Burkholderia pseudomallei & BB 0054 & CP006470.1 Burkholderia pseudomallei \\
Burkholderia pseudomallei & BURK088 & CP006470.1 Burkholderia pseudomallei \\
Burkholderia pseudomallei & BURK099 & CP006470.1 Burkholderia pseudomallei \\
Burkholderia pseudomallei & NR-9320 & CP006470.1 Burkholderia pseudomallei
\end{tabular}

Outcome of pyrosequencing results compared against the NCBI database for alignment matches for either B. mallei or B. pseudomallei. Only the first match for each pyrosequence output is listed in the table. No sequences were obtained in any tests with B. cepacia or other bacterial strains and therefore no blastsearches were performed.

TABLE 4: Results of PCR and pyrosequencing assays for B. mallei and B. pseudomallei.

\begin{tabular}{|c|c|c|c|c|}
\hline \multirow{2}{*}{ Organism name (number) } & \multicolumn{2}{|c|}{ B. mallei Assays } & \multicolumn{2}{|c|}{ B. pseudomallei Assays } \\
\hline & PCR & Pyrosequence & PCR & Pyrosequence \\
\hline B. mallei (10) & + & PASS & - & FAIL \\
\hline B. pseudomallei (10) & - & FAIL & + & PASS \\
\hline B. cepacia (15) & - & FAIL & - & FAIL \\
\hline Burkholderia species (8) & - & FAIL & - & FAIL \\
\hline \multicolumn{5}{|l|}{ Other Bacteria (17) } \\
\hline Gram negative strains (10) & - & FAIL & - & FAIL \\
\hline Gram positive strains $(7)$ & - & FAIL & - & FAIL \\
\hline
\end{tabular}

“+” denotes a positive result or amplicon was produced.

"-" denotes a negative results.

"PASS" denotes the pyrosequencing assay produced a reliable sequence.

"Fail" denotes the pyrosequencing assay produced no reliable sequence or no sequence at all.

a new method for detection and differentiation based on single gene target pyrosequencing. Pyrosequencing is alternative sequencing method, which provides real-time read-out, that is, highly suitable for sequencing short stretches of DNA [36]. Other key advantages of pyrosequencing include simple frequency data and the ability to generate sequence signals immediately downstream of the primer. Additionally, both sample preparation and single-strand DNA processing are relatively rapid [37]. Furthermore, pyrosequencing eliminates the need for labeled primers, dNTPs, or gel electrophoresis. We chose pyrosequencing as our target platform because it requires the least amount of sample manipulation, while still providing real-time read-out results that are highly suitable for sequencing short stretches of DNA ( $\leq 40$ nucleotides). However, the target sequences and the assays described in this paper can be adapted to standard and real-time PCR in those laboratories that do not possess a pyrosequencer. In our experience pyrosequencing is cost-effective in testing samples when compared to qPCR, standard PCR, and dideoxynucleotide chain-terminating sequencing methods. Cost analysis was not in the scope of our paper but could be addressed by other researchers.

Clinically, when infection is suspected and before any treatment options can be explored proper pathogen identification is essential. This ensures that both the proper treatment and safety protocols are followed to protect both patient and 
healthcare practitioner. We proposed an effective method of detection and positive identification of $B$. malle $i$ and $B$. pseudomallei based upon the overall genomic plasticity and multiple IS elements separating $B$. mallei from $B$. pseudomallei $[32,38]$. The IS407A insertion that causes the fliP gene truncation, inversion, and subsequent loss of flagella functionality in $B$. mallei provided the target for reliable identification of both species. Our pyrosequencing assays targeted a short nucleotide sequence that encompassed both the inverted $f$ liP sequence as well as the IS407A sequence. As this sequence is unique to $B$. mallei it was our assertion that this sequence should provide $100 \%$ identification of $B$. mallei. Examination of DNA from ten unique $B$. mallei isolates validated this claim. The fully functional, nontruncated, noninverted fliP gene of $B$. pseudomallei also provided a reliable target for identification. We focused on the region of the gene where the IS407A insertion sequence apparently was introduced to create the $B$. mallei fliP/IS407A interface.

The Burkholderia species contain several genes including fliP that contribute to the biosynthesis and function of the flagellar organelle [39]. While the fliP gene is present throughout the various Burkholderia species, minor sequence differences enabled us to employ specific targeting. B. thailandensis is genetically and phenotypically the closest neighbor to both B. mallei and B. pseudomallei [40]. However, $B$. thailandensis is avirulent towards humans and animals and therefore only three were examined in this study. B. cepacia, (a common opportunistic pathogen) is also genetically related to $B$. mallei and B. pseudomalle $i$ as well as familiar to clinicians and laboratorians in the United States. In addition, B. cepacia carries a version of the fliP gene and has been mistaken for B. pseudomallei [19]. Thus we added more B. cepacia isolates to our examination of the B. pseudomallei assay. $B$. cepacia does not contain any IS element in its fliP gene and thus we predicted that none of the DNA from these isolates would generate positive results with the $B$. mallei primers. The results validated our prediction. The $B$. cepacia DNA also produced negative results in the assays with the B. pseudomallei primers even though the B. cepacia fliP gene is fully functional. Alignment comparison between $B$. pseudomallei (NCBI GenBank Accession \#:NC_009076) and B. cepacia fliP gene resulted in up to $88 \%$ alignment matches (NCBI sequence alignment). Disruption of the fliP gene gives $B$. mallei its distinct nonmotile phenotype, while the differences between $B$. cepacia and $B$. pseudomalle $i$ fliP genes provide genotypic targeting while still retaining their motility phenotype. While the B. cepacia fli $\mathrm{P}$ is fully functional, the sensitivity of pyrosequencing enables the exploitation of even a $12 \%$ difference because the oligonucleotides used in our pyrosequencing assay had low homology with the $B$. cepacia fliP sequence. It is only through sequencing that one can definitively differentiate between $B$. pseudomallei and $B$ cepacia. Our detection assays can enable the laboratory to identify an isolate as either B. mallei or B. pseudomalle $i$ with the confidence of eliminating false positives with $B$. cepacia. If positive for either select agent, the laboratory can immediately send the isolate to a Laboratory Reference Network (LRN) laboratory where it can be further examined and the CDC contacted if necessary. Although B. cepacia is not a focus of LRN laboratories, it is still a serious concern for the clinical laboratory and clinicians. Therefore a new pyrosequencing assay to reliably and consistently identify $B$. cepacia is being explored by our laboratory.

To date, we have not yet identified in the literature any B. mallei strains that do not have the IS407A element disrupting the fliP gene. Historically, this interruption with the subsequent rearrangement of the gene partial sequences' orientation and loss of genetic material has been one feature that distinguishes the B. mallei species from B. pseudomallei. Additionally, the element seems to be stable in $B$. mallei strains although it can theoretically move. Researchers have suggested that $B$. mallei evolved from a sole strain of $B$. pseudomallei and that the genome is "closed" and in "an evolutionary bottleneck in the mammalian host" that offered no opportunity for new genes [41]. It would be very interesting to see if in the future any $B$. mallei isolates have lost the IS element adjacent to the partial fliP gene sequence. If and when that occurs new assays can be designed using the partial fliP sequence and whatever DNA sequence remains after the exit of the IS element.

With new detection assays there is always a concern that since many are developed using pure culture they are susceptible to variable results when challenged with mixed cultures. Clinical samples are routinely cultured in nutrient rich media to identify pathogens and by custom and necessity to isolate each potential pathogen. Our study is designed for usage in clinical applications after the organism has been isolated from a clinical sample such as blood, skin, or deep tissue wound and the organism is not yet identified. So the fact that our assay was developed using pure cultures should have no negative impact on future applications. However, in the future, we plan to examine the possibility of direct detection straight from a clinical sample without using culturing and to test mixtures of bacteria, samples, and specimens such as swamp and irrigation waters, wounds, and sputum to explore if our assays can be utilized without the necessity of culturing to isolate the potential pathogen.

Although PCR based assays are prized for their high sensitivity this can also be a potential drawback. One of the primary drawbacks of PCR assay is "carry-over" products resulting from subsequent PCR runs which then become contaminants producing false positives $[42,43]$. Pyrosequencing requires no initial PCR amplification or subsequent post PCR manipulation. The target sample DNA can be utilized directly as the template. Here we performed PCR only as an experimental validation step in the beginning of the study and later switched to extracted genomic DNA as template. The ability of pyrosequencing to identify short nucleotide sequences gives our assays specificity vital to the successful identification and differentiation of the two Burkholderia species. It is important to note that this assay is a starting point which will require further validation with the necessary cost analysis. In particular as both B. mallei and B. pseudomallei are Tier 1 designees access to multiple strains of DNA is strictly limited. Thus we could not test more isolates as would be required by the CDC. However, we are confident that this work is a starting point that focuses on a single gene as a target 
and can be useful to other researchers whether in the clinical or basic research laboratory.

\section{Conflict of Interests}

The authors declare that there is no conflict of interests regarding the publication of this paper.

\section{Acknowledgments}

The authors wish to thank Ms. Sue Swanzey, MS from the University of Washington Medical Center in Seattle, WA, for generously donating Burkholderia cepacia isolates. The B. mallei and B. pseudomallei were obtained from the NIH Biodefense and Emerging Infections Research Resource's Repository, NIAID, NIH, with the gracious aid of Dr. Andrew Cannons of the Florida Department of Health, Tampa, FL. This work was supported by the United States Department of Defense, DOD Contract Number W911SR-11-C-0017.

\section{References}

[1] I. Janse, R. A. Hamidjaja, A. C. A. Hendriks, and B. J. van Rotterdam, "Multiplex qPCR for reliable detection and differentiation of Burkholderia mallei and Burkholderia pseudomallei," BMC Infectious Diseases, vol. 13, no. 1, article 86, 2013.

[2] S. J. Peacock, H. P. Schweizer, D. A. B. Dance et al., "Management of accidental laboratory exposure to Burkholderia pseudomallei and B. mallei," Emerging Infectious Diseases, vol. 14, no. 7, article e2, 2008.

[3] E. B. Silva and S. W. Dow, "Development of Burkholderia mallei and pseudomallei vaccines," Frontiers in Cellular and Infection Microbiology, vol. 3, p. 10, 2013.

[4] G. C. Whitlock, D. M. Estes, and A. G. Torres, "Glanders: off to the races with Burkholderia mallei," FEMS Microbiology Letters, vol. 277, no. 2, pp. 115-122, 2007.

[5] M. Wheelis, "First shots fired in biological warfare," Nature, vol. 395, article 213, 1998.

[6] L. D. Rotz, A. S. Khan, S. R. Lillibridge, S. M. Ostroff, and J. M. Hughes, "Public health assessment of potential biological terrorism agents," Emerging Infectious Diseases, vol. 8, no. 2, pp. 225-230, 2002.

[7] B. J. Currie, D. A. B. Dance, and A. C. Cheng, "The global distribution of Burkholderia pseudomallei and melioidosis: an update," Transactions of the Royal Society of Tropical Medicine and Hygiene, vol. 102, no. 1, pp. S1-S4, 2008.

[8] V. Ngauy, Y. Lemeshev, L. Sadkowski, and G. Crawford, "Cutaneous melioidosis in a man who was taken as a prisoner of war by the Japanese during World War II," Journal of Clinical Microbiology, vol. 43, no. 2, pp. 970-972, 2005.

[9] K. C. Kronmann, A. A. Truett, B. R. Hale, and N. F. CrumCianflone, "Melioidosis after brief exposure: a serologic survey in US marines," The American Journal of Tropical Medicine and Hygiene, vol. 80, no. 2, pp. 182-184, 2009.

[10] Centers for Disease Control and Prevention, "Imported melioidosis-south Florida, 2005," Morbidity and Mortality Weekly Report (MMWR), vol. 55, no. 32, pp. 873-876, 2006.

[11] S. E. Dorman, V. J. Gill, J. I. Gallin, and S. M. Holland, "Burkholderia pseudomallei infection in a Puerto Rican patient with chronic granulomatous disease: case report and review of occurrences in the Americas," Clinical Infectious Diseases, vol. 26, no. 4, pp. 889-894, 1998.

[12] B. Christenson, Z. Fuxench, J. A. Morales, R. A. Suárez-Villamil, and L. M. Souchet, "Severe community-acquired pneumonia and sepsis caused by Burkholderia pseudomallei associated with flooding in Puerto Rico," Boletin de la Asociacion Medica de Puerto Rico, vol. 95, no. 6, pp. 17-20, 2003.

[13] D. M. Corral, A. L. Coates, Y. C. W. Yau et al., "Burkholderia pseudomallei infection in a cystic fibrosis patient from the Caribbean: a case report," Canadian Respiratory Journal, vol. 15, no. 5, pp. 237-239, 2008.

[14] D. M. Engelthaler, J. Bowers, J. A. Schupp et al., "Molecular investigations of a locally acquired case of melioidosis in Southern AZ, USA," PLoS Neglected Tropical Diseases, vol. 5, no. 10, Article ID e1347, 2011.

[15] D. B. Rolim, M. F. G. Rocha, R. S. N. Brilhante et al., "Environmental isolates of Burkholderia pseudomallei in Ceara State, Northeastern Brazil," Applied and Environmental Microbiology, vol. 75, no. 4, p. 1215, 2009.

[16] Centers for Disease Control and Prevention, "Laboratory exposure to Burkholderia pseudomallei-Los Angeles, California, 2003," MMWR Morbidity and Mortality Weekly Report, vol. 53, no. 42, pp. 988-990, 2004.

[17] K. Vidyalakshmi, M. Chakrapani, B. Shrikala, S. Damodar, S. Lipika, and S. Vishal, "Tuberculosis mimicked by melioidosis," International Journal of Tuberculosis and Lung Disease, vol. 12, no. 10, pp. 1209-1215, 2008.

[18] T. J. Inglis and A. Q. Sousa, "The public health implications of melioidosis," Brazilian Journal of Infectious Diseases, vol. 13, no. 1, pp. 59-66, 2009.

[19] C. Weissert, G. Dollenmaier, P. Rafeiner, J. Riehm, and D. Schultze, "Burkholderia pseudomallei misidentified by automated system," Emerging Infectious Diseases, vol. 15, no. 11, pp. 1799-1801, 2009.

[20] S. A. Batra, S. Krupanidhi, and U. Tuteja, "A sensitive \& specific multiplex PCR assay for simultaneous detection of Bacillus anthracis, Yersinia pestis, Burkholderia pseudomallei \& Brucella species," Indian Journal of Medical Research, vol. 138, no. 1, pp. 111-116, 2013.

[21] T. J. J. Inglis, D. Chiang, G. S. H. Lee, and L. Chor-Kiang, "Potential misidentification of Burkholderia pseudomallei by API 20NE," Pathology, vol. 30, no. 1, pp. 62-64, 1998.

[22] M. B. Glass and T. Popovic, "Preliminary evaluation of the API 20NE and RapID NF plus systems for rapid identification of Burkholderia pseudomallei and B. mallei," Journal of Clinical Microbiology, vol. 43, no. 1, pp. 479-483, 2005.

[23] A. J. Brent, P. C. Matthews, D. A. Dance, T. L. Pitt, and R. Handy, "Misdiagnosing melioidosis," Emerging Infectious Diseases, vol. 13, no. 2, pp. 349-351, 2007.

[24] T. J. J. Inglis, A. Merritt, G. Chidlow, M. Aravena-Roman, and G. Harnett, "Comparison of diagnostic laboratory methods for identification of Burkholderia pseudomallei," Journal of Clinical Microbiology, vol. 43, no. 5, pp. 2201-2206, 2005.

[25] W. Lowe, K. J. March, J. A. Bunnell, OL. K. Neill, and A. R. Robison, "PCR-based methodologies used to detect and differentiate the Burkholderia pseudomallei complex," Current Issues in Intestinal Microbiology, vol. 16, pp. 23-54, 2013.

[26] D. Limmathurotsakul, V. Wuthiekanun, P. Amornchai, G. Wongsuwan, N. P. J. Day, and S. J. Peacock, "Effectiveness of a simplified method for isolation of Burkholderia pseudomallei from soil," Applied and Environmental Microbiology, vol. 78, no. 3, pp. 876-877, 2012. 
[27] J. R. Bowers, D. M. Engelthaler, J. L. Ginther et al., "BurkDiff: a real-time PCR allelic discrimination assay for Burkholderia pseudomallei and B. mallei," PLoS ONE, vol. 5, no. 11, Article ID e15413, 2010.

[28] V. A. Antonov, G. A. Tkachenko, V. V. Altukhova et al., "Molecular identification and typing of Burkholderia pseudomallei and Burkholderia mallei: when is enough enough?" Transactions of the Royal Society of Tropical Medicine and Hygiene, vol. 102, no. 1, pp. S134-S139, 2008.

[29] W. Ruppitsch, A. Stöger, A. Indra et al., "Suitability of partial 16 S ribosomal RNA gene sequence analysis for the identification of dangerous bacterial pathogens," Journal of Applied Microbiology, vol. 102, no. 3, pp. 852-859, 2007.

[30] W. G. Weisburg, S. M. Barns, D. A. Pelletier, and D. J. Lane, "16S ribosomal DNA amplification for phylogenetic study," Journal of Bacteriology, vol. 173, no. 2, pp. 697-703, 1991.

[31] T. G. Lessie, W. Hendrickson, B. D. Manning, and R. Devereux, "Genomic complexity and plasticity of Burkholderia cepacia," FEMS Microbiology Letters, vol. 144, no. 2-3, pp. 117-128, 1996.

[32] H. Song, J. Hwang, H. Yi et al., "The early stage of bacterial genome-reductive evolution in the host," PLoS Pathogens, vol. 6, no. 5, pp. 1-10, 2010.

[33] W. C. Nierman, D. DeShazer, H. S. Kim et al., "Structural flexibility in the Burkholderia mallei genome," Proceedings of the National Academy of Sciences of the United States of America, vol. 101, no. 39, pp. 14246-14251, 2004.

[34] M. Ronaghi, "Pyrosequencing sheds light on DNA sequencing," Genome Research, vol. 11, no. 1, pp. 3-11, 2001.

[35] U.S. Department of Health and Human Services CDC \& $\mathrm{NIH}$, Biosafety in Microbiological and Biomedical Laboratories, Washington, DC, USA, 5th edition, 2007.

[36] E. E. Galyov, P. J. Brett, and D. Deshazer, "Molecular insights into Burkholderia pseudomallei and Burkholderia mallei pathogenesis," Annual Review of Microbiology, vol. 64, pp. 495-517, 2010.

[37] M. D. Fakruddin, A. Chowdhury, M. D. Hossain, S. K. Bin Mannan, and N. R. Mazurmdar, "Pyrosequencing-principles and applications," Life Science, vol. 2, pp. 65-76, 2012.

[38] B. Gharizadeha, M. Akhrasa, N. Nourizadb et al., "Methodological improvements of pyrosequencing technology," Journal of Biotechnology, vol. 124, no. 3, pp. 504-511, 2006.

[39] J. Malakooti, B. Ely, and P. Matsumura, "Molecular characterization, nucleotide sequence, and expression of the fliO, fliP, fliQ, and fliR genes of Escherichia coli," Journal of Bacteriology, vol. 176, no. 1, pp. 189-197, 1994.

[40] F. M. Thibault, E. Valade, and D. R. Vidal, "Identification and discrimination of Burkholderia pseudomallei, B. mallei, and B. thailandensis by real-time PCR targeting type III secretion system genes," Journal of Clinical Microbiology, vol. 42, no. 12, pp. 5871-5874, 2004.

[41] L. Losada, C. M. Ronningf, D. Deshazer et al., "Continuing evolution of Burkholderia mallei through genome reduction and large-scale rearrangements," Genome Biology and Evolution, vol. 2, no. 1, pp. 102-116, 2010.

[42] D. N. Fredricks and D. A. Relman, "Application of polymerase chain reaction to the diagnosis of infectious diseases," Clinical Infectious Diseases, vol. 29, no. 3, pp. 475-488, 1999.

[43] S. Yang and R. E. Rothman, "PCR-based diagnostics for infectious diseases: uses, limitations, and future applications in acute-care settings," The Lancet Infectious Diseases, vol. 4, no. 6, pp. 337-348, 2004. 

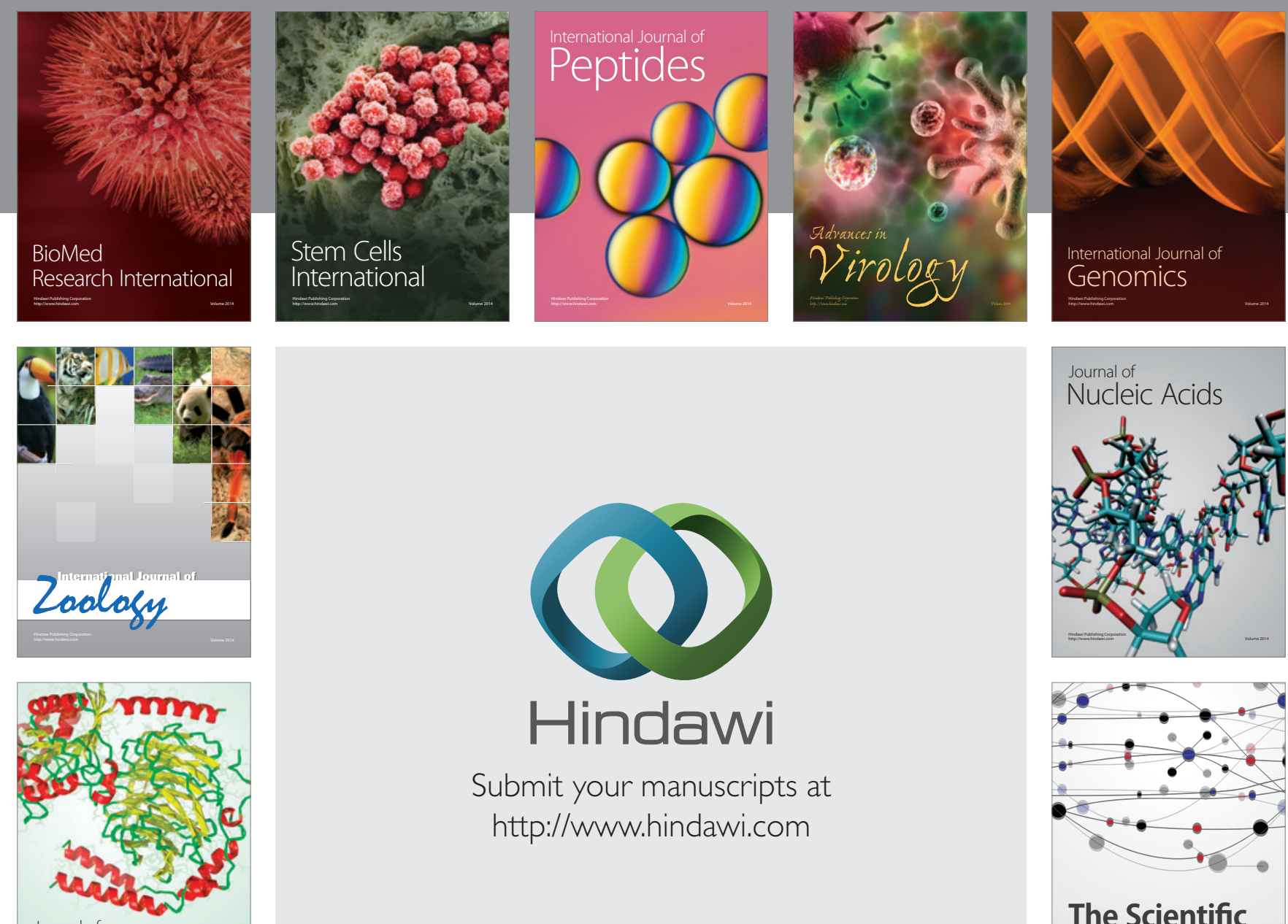

Submit your manuscripts at

http://www.hindawi.com

Journal of
Signal Transduction
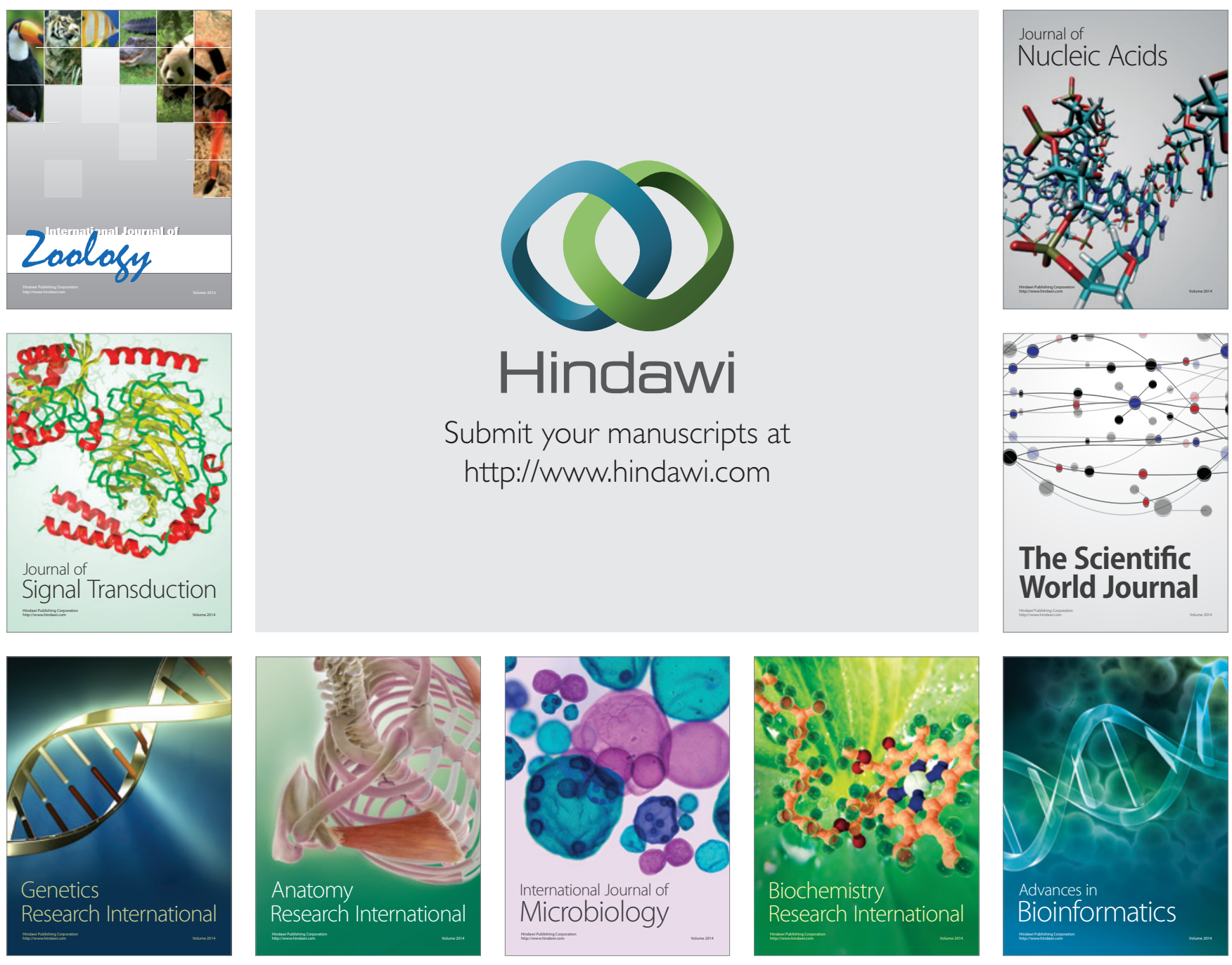

The Scientific World Journal
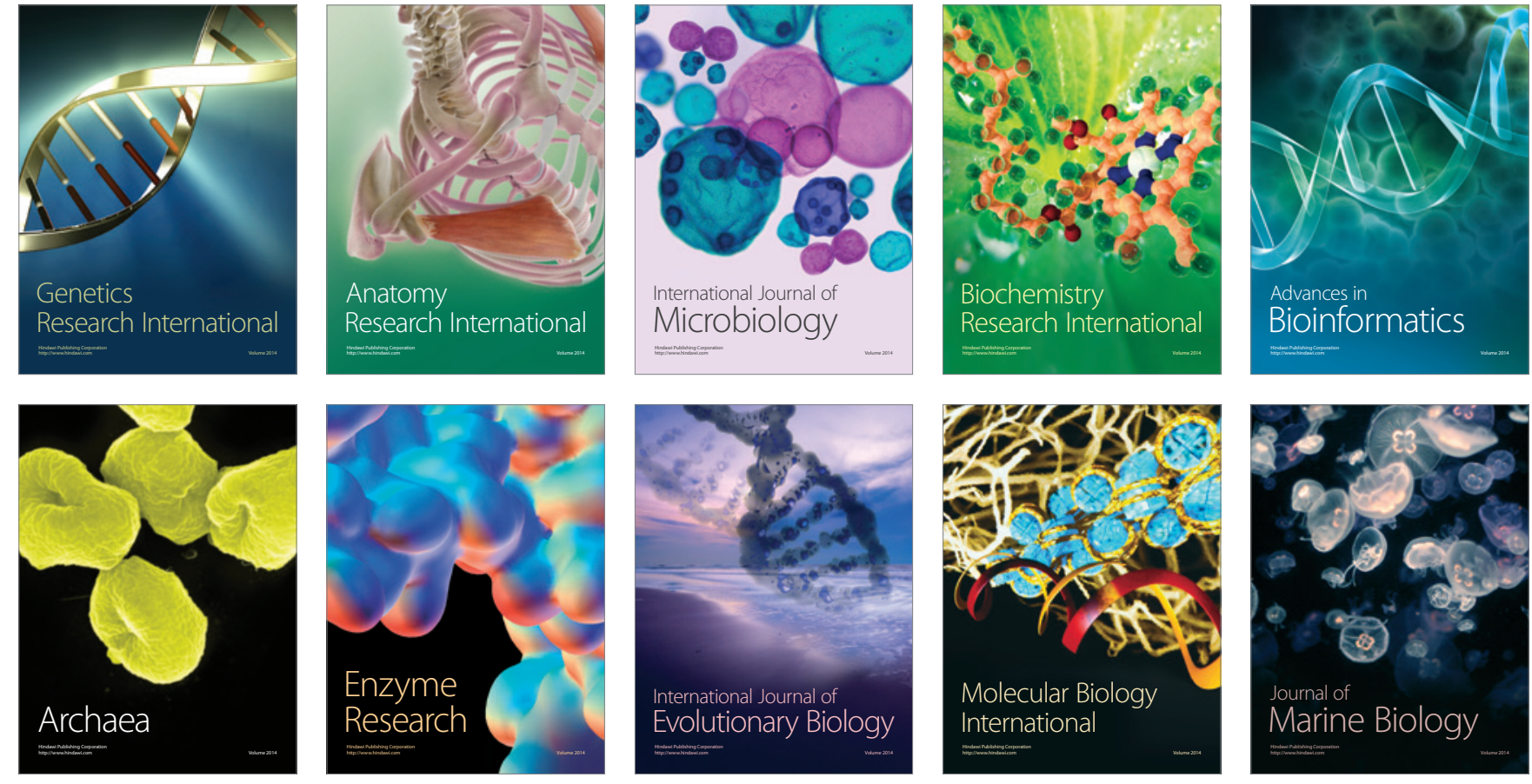